\title{
What is life for science?
}

\author{
Pier Luigi Luisi*i† \\ Professor Emeritus Swiss Federal Institute of Technology (ETH) - Zürich Zentrum - Rämistrasse \\ 101, 8092 Zürich, Switzerland
}

\section{E-mail: Luisidmat.ethz.ch}

The question "what is life for science?" is examined at the light of the theory of autopoiesis by Maturana and Varela, which puts the emphasis on the behaviour of the biological cell. The apparent paradox between the cell auto-maintenance and the myriads of ongoing transformations inside the cell, is solved by the observation that the cell re-generates from within its boundary all components that are going to be consumed away during metabolism. The capability of selfgeneration from within is taken as the most general and most important characteristic of life in general. This process of self-generation is possible thanks to the interaction with the environment, and this brings us to the notion of cognition. All living organisms, including bacteria, are cognitive, in the sense that they are provided with the sensorial tools to recognize and interact with their own specific environment. For humans, cognition takes the form of the five senses, plus the mind, so that the final answer to the question "what is life?" is the trilogy of the autopoietic unit, the environment, and cognition, which are intrinsically linked to each other in a single unity.

The Golden Age of Cataclysmic Variables and Related Objects - III, Golden2015

7-12 September 2015

Palermo, Italy

* Speaker.

${ }^{\dagger}$ A footnote may follow. 


\section{Introduction}

The specification "for science" is important to simplify the content of this article, as the notion of life, in principle, is relevant for quite different domains. For example, in religion it has a very particular connotation, in art it may assume a quite different meaning, and in medicine it may give rise to the still ongoing discussions about euthanasia and the definition of death. Philosophy is of course also a domain in which the question "what is life?" has still to find a definitive answer. One may also think to the famous book by Erwin Schrödinger, "What is life?", and here one must add that even in the general field of life science his question is still open, and in a rather confused way. I cannot review here all the books and monographs in the field, I can refer to my recent book in which hundreds of these references and their history are given (Luisi, 2016). In this article, I will limit myself to present the "biologics" of life, in a way which, in my opinion, represents the best answer to the question of the title. This is the theory of autopoiesis, due to Maturana and Varela $(1980,1984)$ in the middle seventies, and more generally expressed in the literature together with the notion of cognition. This theory is also amply reviewed in my book, and actually these few pages which follow are based on that written text.

\section{A teaching experiment}

But let me introduce the question "what is life?" based on a teaching experiment. Suppose in fact to be the teacher of biology (and/or philosophy) course and present to your student the following table, composed by two different parts: There is a list of living things; and a list of nonliving things. And now you ask your students: what discriminates the one from the other? What is the "thing" which must be present in all the elements of the left list, and cannot be present in any of the elements of the other list?

But you tell them first, that we stage the question in past centuries, when cellular metabolism was not yet known; and DNA had not been discovered. In fact, the question "what is life?" might have been asked in all times, and it would have been possible to give a "scientific" answer centuries ago. Also, you have to say to them that to give GOD as an answer is not valid in this particular exercise.

And here you can begin to collect interesting answers. The first which probably will be given you is "reproduction". All living organism reproduce themselves, in a way or the other. And then you answer: No, to say so, is not correct. Reproduction is a property of life - in order to have reproduction you first have to be alive.

In fact, the difference between "essence" and "property" is essential in life sciences and philosophy. If I ask: what is an airplane? And you answer: it flies. Certainly, a good airplane must fly, otherwise it is good for nothing, but to say "flies" says nothing about the essence of an airplane. Likewise, the expression "the living reproduces itself" is the description of a property, but why there is reproduction? What is the thing that permits reproduction? This is life, so tell me what is life first, and afterwards we will see how this "life" may permit, as one of its properties, reproduction. (Aside from the fact that many living beings are not able to reproduce: in the human kind, all babies, children, and very old ladies and gentlemen, do not reproduce, but are living). Other properties of life may be movement, or energy consumption, or respiration. But it would be 
THE GAME OF THE TWO LISTS

\begin{tabular}{|c|c|}
\hline LIST OF THE LIVING & LIST OF THE NON-LIVING \\
The fly & The radio \\
The tree & The automobile \\
The mule & The robot \\
The baby & The crystal \\
The mushroom & The moon \\
The amoeba & The computer \\
\hline
\end{tabular}

QUESTION: What discriminates the living from the non-living?

IN OTHER WORDS: What is the quality (or qualities) which is present in all members of the "living list" and which is not - and cannot - be present in any of the elements of the "non-living" list?

Figure 1: The lists of living and non-living.

easy to show that such properties are also present, in one way or another, in the second list - an automobile, an electric device, an artificial lung system...

Given the time, this maieutic experiment can bring us to a common, satisfactorily answer. We do not have the space/time here to go on with this exercise, which you can pursue alone in due time. Instead, let me summarize quickly: the common denominator can be seen in the fact that all living things are capable of maintaining their own structure over time thanks to a process of re-generation from within, from their own boundaries.

This is the important main concept of autopoiesis, and to expose it properly, let us leave the class experiment and consider instead, as Maturana and Varela have done, the simplest living entity, which is the biological cell.

It is relevant to add that autopoiesis had its implementation in synthetic chemistry: firstly with a theoretical work by Luisi and Varela (1989), and later with a series of experimental works developed in the Federal Polytechnic of Zürich (e.g. Bachmann, Luisi, and Lang, 1992; Zepik, Blöchliger, and Luisi, 2001).

\section{The cell and the bio-logics of life}

In fact, the life of a cell is the starting point for the development of the ideas of autopoiesis (from the Greek auto, or self, and poiesis, producing) developed by Maturana and Varela (Varela, Maturana \& Uribe 1974; Maturana and Varela, 1980, 1998). Autopoiesis deals with the question "what is life?" and attempts to isolate, above and beyond the diversity of all living organisms, a common denominator that allows for discrimination between the living and the non-living. Au- 
topoiesis is not directly concerned with origin of life; rather, it is an analysis of the living as it is here and now, as the authors say.

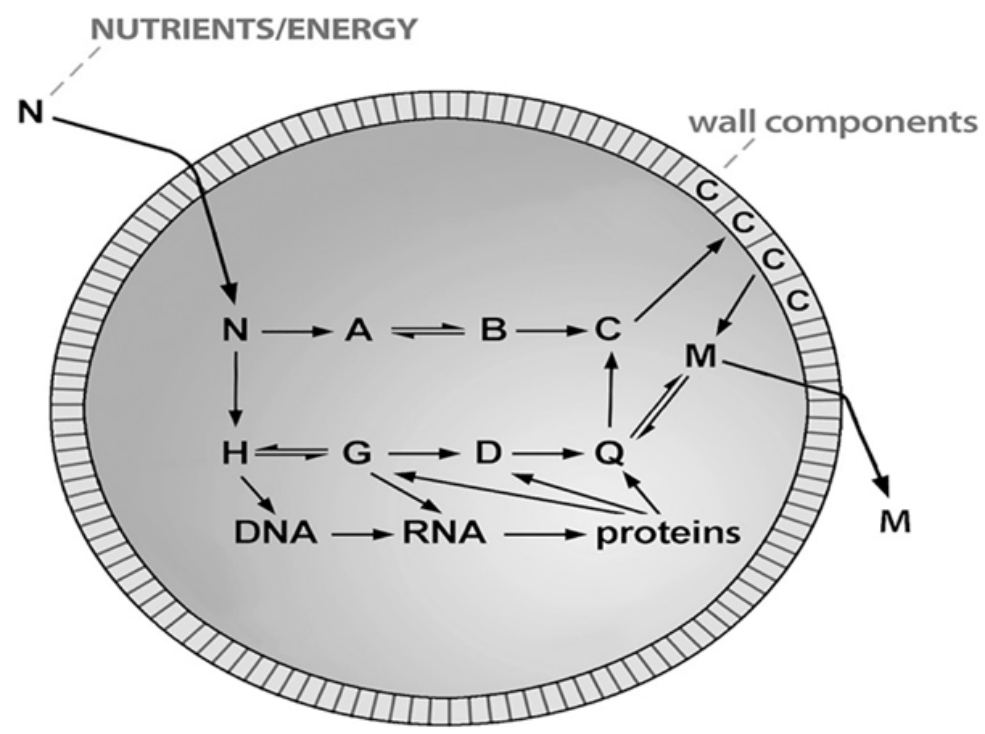

Figure 2: Schematization of the work of a cell as an open system. One important feature is the boundary, which is created by the internal network of reactions (a boundary of its own making). The network of reactions brings about a large series of transformations; however, under homeostatic conditions all material that disappears is generated again by the internal machinery and by transformation of incoming nutrients/energy. Thus, the cell (and, by inference, life) can be seen as a factory engaged in self-maintenance.

We can consider any one of our body's cells; or consider any unicellular bacterium-see Fig. 2, and fig. 3. The first thing one observes from Fig. 2 is the boundary, a semi-permeable, spherical, closed membrane that discriminates the cell from the medium. The term semi- permeable means that certain substances (nutrients and other chemicals) are able to penetrate in the interior, whereas most other chemicals cannot. This is a kind of chemical selection and chemical recognition - a notion that will be later on linked to the term cognition. The point is, that inside this cell there are every second thousands of chemical transformations - the entire metabolism which can be better illustrated on the basis of Fig. 3. The important point is the following: that despite these continuous chemical changes, the liver cell, or that bacterium, remains always the same. The cell's main activity is self-maintenance. How is this self-maintenance possible, in view of all those transformations? The answer is precisely the one we were looking for in our teaching experiment: it is possible because the cell re-generates from its interior all those compounds which are consumed away. The cell is a factory that continuously remakes itself from within. And: what is true for the cell is true for every macroscopic living organism. My hemoglobin disappears every few days, but is re-made by the body's metabolism. The same for my beard, which I cut every day, and which comes again the day after. And actually, most of the cells of our body are renewed every few months - so that we are all new organisms every few months - and yet we can say that we self-maintain. This brings us to the important concept of homeostasis in biology - the dynamic maintenance of constancy over time. Of course, all this is possible because of the uptake of food and energy from 
the environment - all living systems are thermodynamically open systems (superimposed to the homeostasis there is the problem of aging - but this operates, generally, in a different timescale, and we can neglect it here). Some of the details of the metabolism of a cell are illustrated in the example of Fig. 3. From Fig. 3 we can elicit another general feature of life. In fact, looking at Fig. 3, one could ask the question: where is the life of the bacterium localized? The answer, quite general, and very important, is that the life of the bacterium and life in general, cannot be localized. Life is a global system. The living cell - and life in general - as an autonomous system can be seen as a "self without localization" (Varela, Thompson \& Rosch, 1991; Varela, 2000), as there is no single component, or single reaction, that alone is responsible for life. This notion of a collective complex system which has a peculiar functionality, but no "director", no center of command, is an important notion in the modern theory of complexity.

\section{A maze illustrating the chemical reactions that interconvert small molecules in cells.}

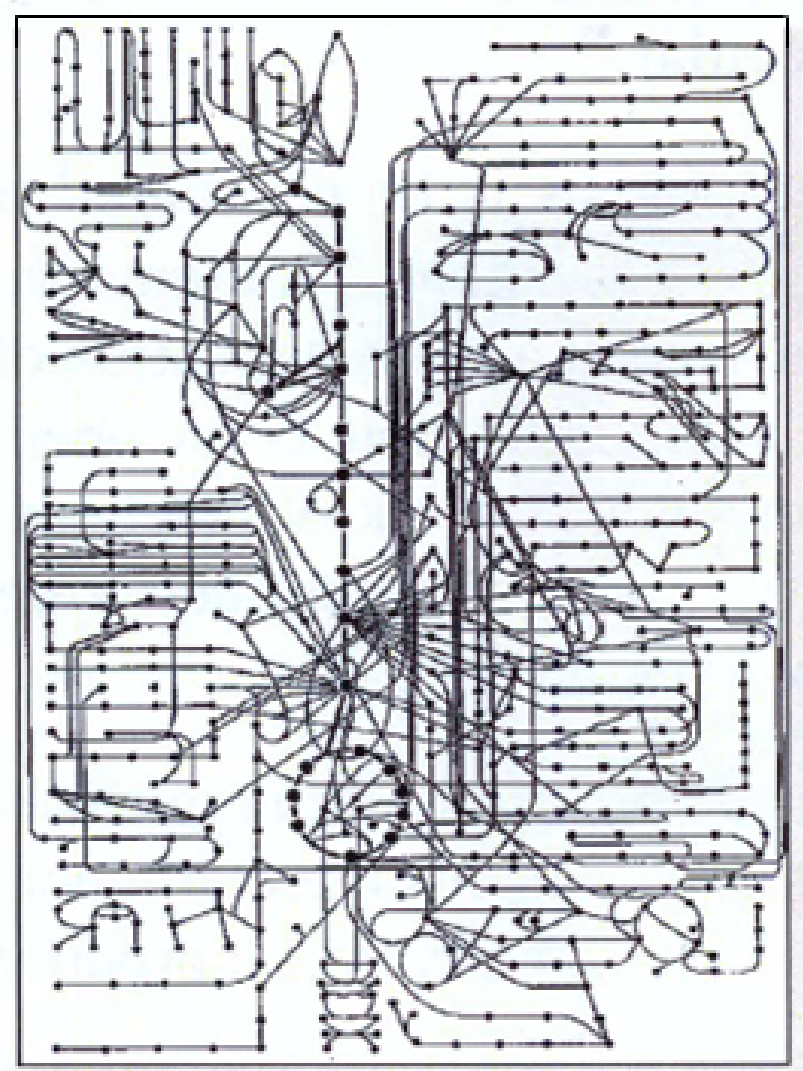

Figure 3: The metabolism (part of) of a cell (E. Coli): each point represents a chemical compound, each line a chemical transformation, which is catalyzed by a specific enzyme.

We mentioned before that reproduction is one of the properties of the living. This is made clear in Fig. 4 where a more detailed representation of cellular autopoiesis is shown.

To be in total agreement with the thinking of Maturana, we can read a strong statement in the conversation with him in the already mentioned book (Luisi 2016), where he says: "... discrete molecular autopoietic systems are living systems, and living system are discrete molecular autopoietic systems, whereby the emphasis, as he adds right away, is on "molecular." 


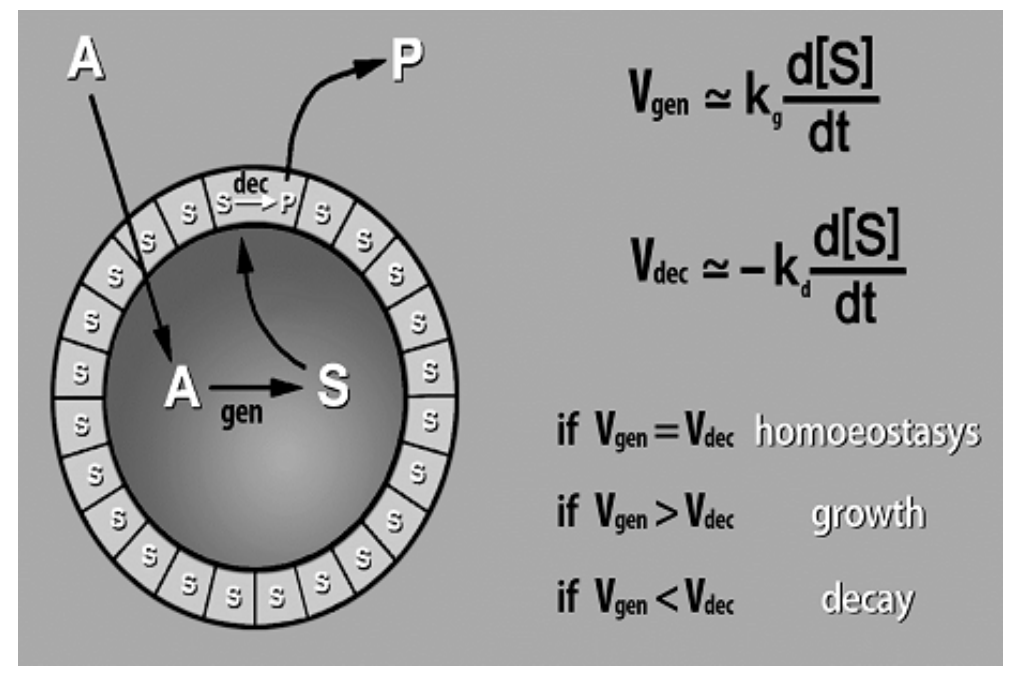

Figure 4: Minimal autopoiesis, with the three modes of cell activity. There are only two schematic processes (in reality each one can be the sum of many of them) namely a rate of generation of chemicals, $V_{\text {gen }}$, then a rate of decomposition, $\mathrm{V}_{\mathrm{dec}}$, and according to their numerical value, the cell can undergo the process of homeostasis, growth (and eventually reproduction), and decay (death).

This is an important point, as autopoiesis is then situated in the realm of chemistry. From these simple, basic observations, Maturana and Varela (often referred to as the Santiago School) arrived at a characterization of living systems based on the autopoietic unit. An autopoietic unit is an open system that is capable of sustaining itself due to an inner network of reactions that regenerate the system's components (Varela, Maturana, \& Uribe, 1974; Maturana and Varela, 1980; Luisi, 1997; Maturana and Varela, 1998; Varela, 2000).

In other words, it can be said that an autopoietic system organizes the production of its own components, so that these components are continuously regenerated and can therefore maintain the very network process that produces them.

It is perhaps pertinent at this point to cite a recent definition by Maturana himself (Poerksen, 2004): "When you regard a living system you always find a network of processes or molecules that interact in such a way as to produce the very network that produced them and that determines its boundary. Such a network I call autopoietic. Whenever you encounter a network whose operations eventually produce itself as a result, you are facing an autopoietic system. It produces itself. The system is open to the input of matter but closed with regard to the dynamics of the relations that generate it." The components organize themselves (auto-organization) in a bounded system, which produces the components that in turn produce the system, and so on. In this way, the blueprint of life obeys a circular logic without an identified beginning or end. Although the system is open from the physical point of view, from an epistemological perspective it has a logical operational closure (Maturana and Varela, 1998; Varela, 2000). This characterizes the system as an autonomous identity that can be defined as auto-referential. It produces its own rules of existence and therefore has a particular type of bio-logical coherence. These internal rules of the system are what make cellular life. This is a descriptive definition of the minimal living system, which does not focus on the structure of the cell's components. It focuses, instead, on the organization of life's basic 
unit, that is, on the invariant functional relations that connect the elementary components into a persistent unity. This also means that autopoiesis is a systemic view, in which the interactions among the components that constitute the essence of the thing are the primary focus.

By so doing, Maturana and Varela propose an operational definition of the basic living system that is very general, for it is able to characterize not only different cellular systems, but rather every kind of system whose structure realizes an autopoietic organization. Here lies the main hypothesis of autopoietic biology, affirming that the definition of the autopoietic system holds true for every living structure: if a physicochemical system is living, then it is autopoietic.

Important are the criteria of autopoiesis: when can we say that a system is autopoietic?

The most general property of an autopoietic system is the capability to generate its own components via a network process that is internal to its boundary. The boundary of the system must be "of its own making," a product of the process of component production. Whether a given system is capable of making its own boundary is the most discriminating criterion by which we recognize an autopoietic system.

More in general, the question of the criteria of autopoiesis is formalized at length, but not always clearly, in the primary literature on autopoiesis. Varela, in his latest book (2000), has simplified these criteria into three basic ones, which can be expressed as 1 . to verify whether the system has a self-produced boundary; 2. Check whether the system encompasses reactions that regenerate the components of the system; 3 . and that this network of reactions is produced by the system itself.

\section{Going into the core of autopoiesis}

We have spoken about the self-maintenance of the cell, or in general about the autopoietic unity. What is it precisely that is maintained? The answer is: the overall cell organization. Here is where the notion of organization acquires its full value. The organization is the invariant of the dynamics of the biological systems; it is the complex of relations that form the identity of the living. The continuous production and conservation of the basic functional relations permit the living to acquire and regenerate its individuality. All this implies that the attention is not on the components, but rather on the relations that these components have to satisfy to constitute the integer system. Therefore, autopoiesis, as already said, belongs to the systems view of life (Capra and Luisi, 2014).

The notion of invariance of the relations is what makes autopoiesis a scientifically rigorous description of biological systems, able to distinguish the invariant aspects of the living dynamics (the organizational relations) from the variable ones and to link them to each other. The structure, the concrete unity of the components, is the variable element; it varies from cell to cell, and it also varies during development, but these changes do not affect the invariance of the biological organization.

The invariance of the relationships among the processes that make the components, as already said, is the reflection of a particular characteristic of the biological self-organization: all rules to produce itself (autopoiesis) are within the boundary, as expressed by the notion of organizational closure - a concept introduced already by Piaget (1967). (Nowadays, the term operational closure is being more commonly used.) It is a closed domain of relations, which defines a space in which a concrete system can be realized (Maturana and Varela, 1980). It is such a closure that gives 
the cellular system the capability to specify from within all operations of production, in a closed chain of linked dynamic operations. In other words, all relations of production are coordinated in a system that can be described as a homeostatic system, which maintains the integrity of its own identity through the production of its own components, also under conditions of perturbation.

This link with the outside is indeed another essential facet of the theory of autopoiesis. The main point here is that the outside must be recognized from the inside, it must positively interact with the autopoietic organization of the living itself. This gives rise to a series of notions, which will be sketched briefly in the next section: firstly, the notion of structural coupling, which, describing the biological interaction with the environment, introduces the new idea of cognition, which in turn involves the notion of information and eventually of evolution.

There are several aspects of autopoiesis that cannot be covered in this article. For example, social autopoiesis (the extension of the notion of autopoiesis to social life), chemical autopoiesis (the laboratory synthesis of cellular models of autopoiesis), autopoiesis of higher order (the extension to multicellular organisms and their organization)- all this however can be found in the specialized literature, including my previously cited second edition of "The emergence of life" (Luisi 2016).

Here, I would like to briefly cover the notion of cognition, which is usually directly associated with the notion of autopoiesis, and which has to do with the interaction of the autopoietic structures with the environment.

\section{About cognition}

Along with the question "What is life," there was another question on Maturana's agenda, namely "What is cognition?" In general, it can be said that autopoiesis is concerned with organization, and cognition with the "doing" of the organism in its environment. In investigating the relationship between these two questions, Maturana and Varela arrived at the conclusion that the two notions, life and cognition, are indissolubly linked to each other, in the sense that one cannot exist without the other. The additional strong point is that each living organism is considered to be cognitive - including bacteria.

The view of Maturana and Varela about the interaction of the living with the environment is within a tradition of thought which was already present in the literature, although in different contexts. Thus, the well-known biologist and genetist Lewontin, mentioning that the atmosphere we all breathe was not on Earth before living organisms, he wrote (Lewontin, 1993): ... There is no "environment" in some independent and abstract sense. Just as there is no organism without an environment, there is no environment without an organism. Organisms do not experience environments. They create them. They construct their own environments out of the bits and pieces of the physical and biological world, and they do so by their own activities.

And it is interesting to observe that this way of thinking is not only proper among biologists, but it is also present in certain European philosophers such as Maurice Merleau-Ponty. Consider the following statement by the French author (Merleau-Ponty, 1967): It is the organism itself - according to the proper nature of its receptors, the thresholds of its nerve centers and the movements of the organs - which chooses the stimuli in the physical world to which it will be sensitive. The environment emerges from the world through the actualization or the being of the organism. 
The view of Maturana, in particular, departs from the current literature mostly because of the particular emphasis given to the concept of internal closure, or operational closure, according to which the "doing" of the organism is dominated by the internal organization, and not due to the external input. All that is needed for an ant to be an ant is in its own internal organization, there is no need of information from the outside in order to be an ant-or an elephant. This is not in contradiction with the fact that the living organism is an open system: the point is, that the interaction with the environment - actually the "perturbation" - in order to be accepted, must be consistent with the internal logic of the living being. In other words, the interaction between an autopoietic unit and a given molecule $\mathrm{X}$ is not primarily dictated by the properties of the molecule $\mathrm{X}$, but by the way in which this molecule is "seen" by the living organism.

As Varela puts it (Varela, 2000): There is no particular nutrient value in sugar, except when the bacterium is crossing the sugar gradient and its metabolism utilizes the molecule so as to permit the continuity of its identity.

Actually, the compounds that the living organism extracts from the environment can be seen as something that the organism itself lacks for implementing its life. The interaction with the environment is characterized by what Maturana and Varela call structural determinism, namely it is determined by the internal organization of the living. In turn, as already mentioned, such a structural determinism for each particular organism is due to the tools developed in the biological evolution, and in fact we can see the environment and the living as co-evolving.

This is in practice the complete notion of cognition: the fact that each organism interacts with the environment in a cognitive matter, with and by the tools developed in nature, by evolution, to account for its specificity. Clearly the interaction of the fish with the water is based on a series of "cognitive tools" which are very different from those of the earth worm to find its way of life.

This means that each living organism "sees" the world in its own way, as due to its internal organization. Actually, the two Chilean authors state that each interaction with the environment "brings forth" a world, namely creates a particular vision of the world. This view, as Maturana likes to repeat, puts the notion of objectivity in quotation marks, in parenthesis- as there are as many world as many living entities. The link between autopoiesis and cognition must be completed by the observation that the cognitive interaction brings about a new reality: the environment is created by the living organism - and the living organism is also created by this interaction with the environment - and this through a series of recursive interactions, which in turn have been produced during the mutual co-evolution. In this process of enaction, a term proposed by Varela (2000), or co-emergence, as we can say more generally, the organic living structure and the mechanism of cognition are two faces of the same phenomenon of life (Varela, 2000). Cognition, then, operates at various levels, and as the sophistication of the organisms grows in evolution, so does the "sensorium" (sensorial tools) for the environment, and so does the extent of co-emergence between organisms and environment. Thus, we go from unicellular to multi-cellular organisms, where we can have flagella and light - or sugar - sensitive receptors, to the development of sensitive tentacles in the first aquatic organisms, and up to the higher cognitive functions in fish. In all these cases, the organisms contribute to the "creation" of their environments. For example, the onset of photosynthetic organisms may have indeed created a novel oxygen-rich environment.

The term create may sound forced, but it is not. Consider Figure 5, with the spider "creating" its own web, its own world. Or you can look at the termites, or ant, nest; a bee hive, the beaver' 
construction; and finally, the cities of mankind.
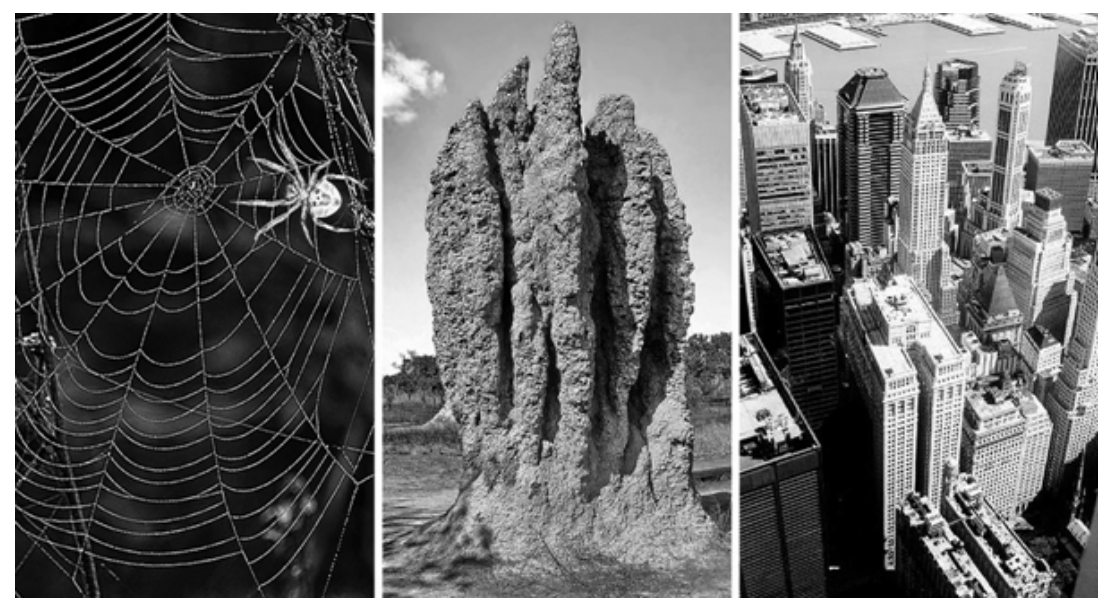

Figure 5: The spider web, the termite nest, and the city of humans as mutual co-emergence of living beings with their own specific environments.

More generally, the emphasis and overall concern here is not to define cognition in terms of an input from the external world acting on the perceiver, but rather to explain cognition and perception in terms of the internal structure (in all its implications, also psychical) of the organism. In this view, autopoiesis and cognition are closely linked. The important feature of both is that they represent a general pattern applicable to all levels of life.

With humans, the sophistication of the sensorial tools acquires a particular level of functional complexity; there are first of all our senses, but there is also the development of the central nervous system and with it, the emergence of mind. However, from the flagella up to the brain, the same basic mechanism is operative: acts of cognition and mutual co-emergence with their own specific environments.

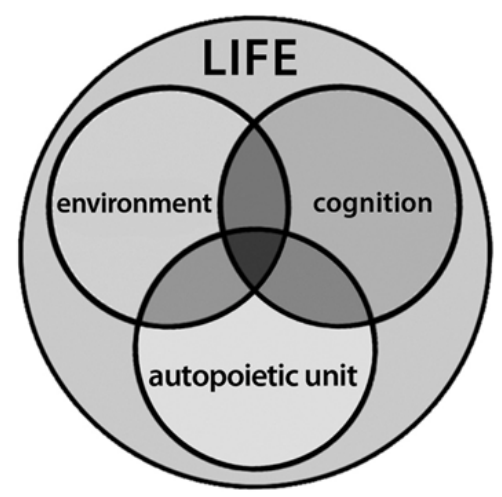

Figure 6: The trilogy of life (from Capra and Luisi, 2014). The organic, living structure interacts with the environment via a cognition sensorium, which is a specific product of its development and evolution. It does not make sense to consider each of these three domains as independent of one another. Life is the synergy of the three domains, as the notion of the "embodied mind" implies. 
At this point, having recognized that life has no meaning without cognition, we need a way to represent the whole of this complex situation. In a first approximation, this can be done with the trilogy shown in Fig. 6.

Here we see life represented not only in terms of the autopoietic unit, but in terms of a trilogy, where the living organic structure (autopoietic unit) interacts cognitively with the environment. As said above, each specific organism has its own cognitive set of sensory apparatus. When one substitutes the term cognition with mind, one arrives at the notion of the "embodied mind".

This notion, proposed by Varela already in the 1990s (Varela, Thompson \& Rosch 1991) is now widely accepted in cognitive science (see also Capra and Luisi, 2014, Chapter 12). It means that it makes no sense to talk about mind in an abstract way. Mind is always present in a bodily structure; and vice versa, in order to have truly living organisms, it must be capable of cognition (the process of knowing). The same holds for human consciousness. Consciousness (at least at the level of sensorial consciousness) is not a transcendent entity but is always manifesting within an organic living structure; the same notion applies as for mind/embodied mind, and embodied consciousness.

\section{Conclusions}

In this way, we have given an answer to the title question, what is life for science (life-science). We have described a design that goes from the cell to the realm of consciousness, while remaining in the realm of biology and without using any transcendent or metaphysical aspects. This entire wide spectrum appears as a product of immanence, namely a construction from within. In closing the present article, we should mention that, at the human level, the interactions between organisms and their environments include both interactions among humans (the domain of the social sciences) and interactions with nature (the domain of ecology).

Acknowledgments I would like to thank Professor Franco Giovannelli for the kind invitation, and the LOC for the hospitality. The editor acknowledges the assistance of James Howarth Beall for his timely review of the paper.

\section{References}

[1] Bachmann, P.A., Luisi, P.L., Lang, J.: 1992, Autocatalytic self-replicating micelles as models for prebiotic structures, Nature, 357, 57-59.

[2] Capra, F., Luisi, P.L.: 2014, The Systems View of Life: A Unifying Vision, Cambridge University Press.

[3] Lewontin, R.C.: 1970, The units of selection, Annu. Rev. Ecol. Syst. 1, 1-18.

[4] Lewontin, R.C.: 1993, The Doctrine of DNA - Biology as an Ideology, Penguin Books.

[5] Luisi, P.L.: 2016, The emergence of life, Second Edition, Cambridge Univ. Press.

[6] Luisi, P.L., Varela, F.J.: 1989, Self-replicating micelles - A chemical version of a minimal autopoietic system, Origins of Life and Evolution of the Biosphere, Volume 19, Issue 6, 633-643.

[7] Maturana, H., Varela, F.: 1980, Autopoiesis and Cognition: The Realization of the Living, Reidel. 
[8] Maturana, H.R., Varela, F.J.: 1998, The Tree of Knowledge, (Based on Revised Edition of 1992). Shambala. (First Edition. 1984, El árbol del conocimiento. Bases biológicas del entendimiento humano. First English Edition. 1987).

[9] Merleau-Ponty, M.: 1967, The Structure of Behaviour, Beacon.

[10] Piaget, J.: 1967, Biologie et connaissance, Gallimard.

[11] Poerksen, B.: 2004, The Certainty of Uncertainty, Dialogues Introducing Constructivism, Imprint Academic.

[12] Varela, F.J.: 1979, Principles of Biological Autonomy, North Holland/Elsevier.

[13] Varela, F.J.: 2000, El Fenómeno de la Vita, Dolmen Ensayo.

[14] Varela, F.J., Maturana, H.R., Uribe, R.B.: 1974, Autopoiesis: the organization of living system, its characterization and a model, Biosystems, 5, 187-196.

[15] Varela, F. J., Thompson, E., Rosch, E.: 1991, The Embodied Mind: Cognitive Science and Human Experience, Cambridge, MA: MIT Press.

[16] Zepik, H.H., Blöchliger, E., Luisi, P.L.: 2001, A Chemical Model of Homeostasis, Angew. Chemie Int. Ed. 40 No.1, 199-202. 